\title{
Síndrome Metabólica em Hipertensos de Cuiabá - MT: Prevalência e Fatores Associados
}

\author{
Metabolic Syndrome in Patients with High Blood Pressure in Cuiabá - Mato Grosso State: Prevalence and \\ Associated Factors

\begin{abstract}
Gilberto Paulo Pereira Franco, Luiz César Nazário Scala, Carlos José Alves, Giovanny Vinícius Araújo de França, Tatiane Cassanelli, Paulo César Brandão Veiga Jardim

Instituto de Saúde Coletiva. Universidade Federal de Mato Grosso, Cuiabá, MT - Brasil
\end{abstract}

\section{Resumo}

Fundamento: A síndrome metabólica (SM) é um agregado de fatores predisponentes para doenças cardiovasculares e diabete melito, cujas características epidemiológicas são insuficientemente conhecidas nos níveis regional e nacional.

Objetivo: Estimar a prevalência de SM e fatores associados em uma amostra de hipertensos da área urbana de Cuiabá - MT.

Métodos: Estudo de corte transversal (maio a novembro de 2007) em amostra de 120 hipertensos (com 20 anos ou mais), pareados por gênero e selecionados por amostragem sistemática de uma população fonte de 567 hipertensos de Cuiabá. Todos os selecionados responderam a um inquérito em domicílio para obtenção de dados sócio-demográficos e hábitos de vida. Foram medidos: pressão arterial; índice de massa corpórea (IMC); circunferências da cintura e quadril; glicemia; insulinemia; lípides séricos; cálculo do índice de homeostase da resistência insulínica (HOMA); proteína C-reativa; ácido úrico e fibrinogênio. O critério para hipertensão adotado foi: média da PAS $\geq 140 \mathrm{mmHg}$ e/ou PAD $\geq 90 \mathrm{mmHg}$, para síndrome metabólica segundo a I Diretriz Brasileira de Síndrome Metabólica e NCEP-ATP III.

Resultados: Foram analisados 120 hipertensos (60 mulheres), com média de idade de 58,3 \pm 12,6 anos. Observou-se prevalência de SM de 70,8\% (IC95\% 61,8-78,8), com predomínio entre as mulheres $(81,7 \%$ vs. $60,0 \% ; p=0,009)$, sem diferenças entre adultos $(71,4 \%)$ e idosos $(70,2 \%)$. A análise de regressão múltipla revelou uma associação positiva entre a SM e o IMC $\geq 25 \mathrm{~kg} / \mathrm{m}^{2}$, a resistência insulínica e algum antecedente familiar de hipertensão.

Conclusão: Observou-se uma elevada prevalência de SM entre hipertensos de Cuiabá, associada significativamente ao IMC $>25 \mathrm{~kg} / \mathrm{m}^{2}$, à resistência insulínica (Índice HOMA) e, em especial, a uma história familiar de hipertensão. Estes resultados sugerem o aprofundamento deste assunto através de novos estudos. (Arq Bras Cardiol 2009;92(6):472-478)

Palavras-chave: Síndrome metabólica, hipertensão, fatores de risco, Cuiabá (MT), Brasil.

\section{Summary}

Background: Metabolic Syndrome (MS) is a cluster of predisposing factors for cardiovascular diseases and diabetes mellitus, whose epidemiological characteristics are poorly known at regional and national levels.

Objective: To estimate the prevalence of MS and its associated factors in a sample of patients with high blood pressure in the urban area of Cuiabá, Mato Grosso State.

Methods: This was a cross-sectional study (May to November 2007) in a sample of 120 patients with high blood pressure (aged $\geq 20$ years), paired by gender and selected by the systematic sampling of a source population of 567 patients with high blood pressure in Cuiabá. All patients answered to home inquiries to provide sociodemographic and life habits data. The following measurements were taken: blood pressure; body mass index (BMI); waist and hip circumferences; plasma glucose, insulin, and lipid levels; homeostasis model assessment-estimated insulin resistance (HOMA); C-reactive protein, uric acid and fibrinogen levels. High blood pressure criterion: average systolic blood pressure $\geq$ $140 \mathrm{mmHg}$ and/or diastolic blood pressure $\geq 90 \mathrm{mmHg}$; and Metabolic Syndrome diagnosis according to the I Brazilian Directive for Metabolic Syndrome and the NCEP-ATP III criteria.

Results: 120 patients (60 women), with high blood pressure and an average age of $58.3 \pm 12.6$ years, were analyzed. We found a MS prevalence of $70.8 \%$ (95\% Cl 61.8 to 78.8$)$, predominantly among women $(81.7 \%$ vs. $60.0 \% ; p=0.009)$, with no difference between adults (71.4\%) and elderly patients (70.2\%). The multiple regression analysis showed a positive association between $\mathrm{MS}$ and $\mathrm{BMI} \geq 25 \mathrm{~kg} / \mathrm{m}^{2}$, insulin resistance and family history of high blood pressure.

Conclusion: A high prevalence of MS was observed among patients with high blood pressure living in Cuiabá, with a significant association with BMI > $25 \mathrm{~kg} / \mathrm{m}^{2}$, insulin resistance (HOMA index) and, especially, a family history of high blood pressure. These results suggest the need for deeper studies on this subject. (Arq Bras Cardiol 2009;92(6):437-442)

Key words: Metabolic syndrome; hypertension; risk factors; Cuiaba (MT); Brazil.

Full texts in English - http://www.arquivosonline.com.br

Correspondência: Gilberto Paulo Pereira Franco •

Rua Estocolmo, 300 - Residencial Alphagarden - Parque Rodoviário - 78048-580, Cuiabá, MT - Brasil

E-mail: gppf@terra.com.br

Artigo recebido em 31/07/2008; revisado recebido em 15/10/2008; aceito em 22/10/2008. 


\section{Introdução}

A síndrome metabólica (SM) é uma condição clínica em que coexistem alguns fatores de risco cardiovascular em maior prevalência do que na população geral. A partir da descrição inicial e dos estudos realizados por Reaven (1988), consolidouse o elo entre um conjunto de anormalidades metabólicas e hemodinâmicas em um mesmo indivíduo, com uma mesma base fisiopatológica: a resistência à insulina ${ }^{1}$. Estes fatores são: pressão arterial $(\mathrm{PA}) \geq 130 \times 85 \mathrm{mmHg}$, resistência à insulina, alterações no metabolismo da glicose (glicemia de jejum $>100 \mathrm{mg} / \mathrm{dl}$, ou diabete tipo 2), obesidade central (circunferência da cintura $\geq 102 \mathrm{~cm}$ no homem e $\geq 88$ $\mathrm{cm}$ na mulher), alterações lipídicas (HDL $<40 \mathrm{mg} / \mathrm{dl}$ no homem ou $<50 \mathrm{mg} / \mathrm{dl}$ na mulher, triglicérides $>150 \mathrm{mg} / \mathrm{dl}$ ), distúrbios da coagulação sanguínea, estado pró-inflamatório e microalbuminúria ${ }^{2-4}$. O termo síndrome metabólica, proposto pela Organização Mundial de Saúde (OMS) em 1998, contribuiu para reformular a compreensão de que vários fatores de risco cardiovascular, não associados por acaso, atuam de forma global para o desenvolvimento da aterosclerose. A denominação SM, a mais aceita atualmente, foi precedida por outras como: síndrome de resistência insulínica, síndrome plurimetabólica, síndrome X e quarteto mortal. A SM pode aumentar a mortalidade geral em cerca de 1,5 vez, e a cardiovascular em, aproximadamente, 2,5 vezes $^{3}$. A hipertensão arterial (HA), importante componente da SM, que ocorre com maior prevalência na população de obesos e diabéticos tipo 2, constitui-se na principal causa da mortalidade cardiovascular precoce em todo o mundo, especialmente o acidente vascular encefálico (AVE) $)^{5,6}$. A prevalência de SM é elevada e variável (13,7\% a 39\%,), com predomínio, em alguns estudos, entre as mulheres ${ }^{7-10}$. Estudos epidemiológicos confirmaram sua ocorrência em diferentes grupos étnicos: europeus; afro-americanos; mexicanoamericanos; asiáticos; chineses; aborígenes australianos; polinésios e micronésios ${ }^{11}$. A existência de um elo molecular (gene ou genes) unificador da fisiopatologia da SM, sugerida por diversos estudos, carece de provas mais consistentes ${ }^{11}$. No Brasil, por exemplo, observa-se um crescente interesse em se conhecer as características epidemiológicas desta síndrome ${ }^{4}$. O objetivo deste estudo é estimar a prevalência da SM, e sua associação com alguns fatores de risco, em hipertensos de Cuiabá, no Centro-Oeste brasileiro.

\section{Métodos}

Foi feito um estudo de corte transversal usando o banco de dados do "Estudo da Prevalência e do Conhecimento da Hipertensão Arterial e alguns Fatores de Risco em uma Região do Brasil", realizado pelo Instituto de Saúde Coletiva e pela Faculdade de Ciências Médicas (Universidade Federal de Mato Grosso - UFMT), em cooperação com a Universidade Federal de Goiás - UFG (Liga de Hipertensão Arterial e Faculdade de Medicina). A população fonte foi constituída por todos os indivíduos $(n=567)$ classificados como hipertensos, em estudo anterior sobre prevalência de hipertensão em CuiabáMT, realizado de fevereiro de 2003 a agosto de 2004 ${ }^{12}$. A prevalência de HA detectada nesse estudo foi estimada em $33,4 \%$. Através de amostragem sistemática, que considerou a densidade populacional das áreas, calculou-se uma população amostral de 120 indivíduos, pareados por gênero, com idade igual ou superior a 20 anos. Foram excluídos os indivíduos acamados, os inválidos, as gestantes, as lactantes e as empregadas domésticas. Em domicílio, os 120 participantes responderam a um questionário pré-testado sobre dados sóciodemográficos; de hábitos de vida (consumo de sal e gordura, atividade física, hábitos tabágico e alcoólico); conhecimento, tratamento e controle de $\mathrm{HA}$; antecedentes familiares de $\mathrm{HA}$; doença arterial coronariana (DAC) e AVE. Foram realizadas três medidas da pressão arterial, com intervalo de 10 minutos entre cada uma, considerando-se a média das duas últimas medidas, classificada segundo as V Diretrizes Brasileiras de Hipertensão Arterial $(2006)^{13}$. Foi utilizado um esfigmomanômetro eletrônico, automático, oscilométrico (OMRON-HEM 705 $\mathrm{CP})$, validado e recomendado por instituições internacionais competentes para estudos epidemiológicos ${ }^{14}$. As medidas foram efetuadas com o entrevistado: sentado; com os pés apoiados no chão; o braço esquerdo relaxado, apoiado sobre mesa e à altura do coração; a palma da mão voltada para cima; a bexiga vazia; sem ter praticado exercícios moderados ou pesados, fumado ou ingerido bebida alcoólica nos 30 minutos antecedentes às medidas. Utilizou-se uma braçadeira compatível com a circunferência do braço. A estatura e o peso foram medidos com estadiômetro Seca Bodymeter 208 e balança Plenna Lithium Digital Mea 08128, para cálculo do índice de massa corpórea (IMC) e classificação do estado nutricional do indivíduo ${ }^{15}$. A circunferência da cintura (CC) foi medida com fita metálica inextensível Cardiomed sobre a pele, ajustada ao corpo, tomando como parâmetro a parte mais estreita do tronco, entre o tórax e os quadris. Foi calculada também a relação cintura-quadril (RCQ). A CC foi classificada em aumentada ou muito aumentada segundo os critérios da OMS (1998) e a relação cintura-quadril (RCQ) em aumentada (>1 para homens e 0,85 para mulheres) ${ }^{15}$.

Foi então colhida uma amostra de sangue venoso (após 12 horas de jejum) por punção da fossa antecubital, para determinação de: glicemia de jejum, lípides séricos, uricemia (método enzimático), insulinemia (quimioluminescência), proteína C-reativa (PCR) e fibrinogênio (turbidimetria). A partir da glicemia e da insulinemia, calculou-se o índice de homeostase da resistência insulínica (HOMA), através da fórmula: glicose (mg/dl) x 0,0555 x insulina / 22,4. Também foram adotados o ponto de corte de 2,71 para definição de resistência insulínica ${ }^{16}$ e os critérios das IV Diretrizes Brasileiras Sobre Dislipidemias e Prevenção da Aterosclerose para os lípides séricos ${ }^{17}$.

\section{Critérios de anormalidades e definições}

Como variável dependente, tivemos a síndrome metabólica, critério da "I Diretriz Brasileira de Diagnóstico e Tratamento da Síndrome Metabólica" (IDBSM) ${ }^{3}$, que utilizou os critérios do National Cholesterol Evaluation Program for Adult Treatment Panel III (NCEP-ATP III) ${ }^{18}$.

Já as variáveis independentes foram: hipertensão arterial, critério das "V Diretrizes Brasileiras de Hipertensão Arterial"13; pressão arterial sistólica (PAS) $\geq 140 \mathrm{mmHg}$ e/ou pressão arterial diastólica (PAD) $90 \mathrm{mmHg}$. Sobrepeso, definido pelo IMC (peso/altura $\left.{ }^{2}\right)\left(>25 \mathrm{~kg} / \mathrm{m}^{2}\right.$ e $\left.<30 \mathrm{~kg} / \mathrm{m}^{2}\right)$ e obesidade 
( $>30 \mathrm{~kg} / \mathrm{m}^{2}$ de superfície corporal; obesidade central, definida pelos pontos de corte da CC em $102 \mathrm{~cm}$ para homens e 88 $\mathrm{cm}$ para mulheres); lípides séricos (jejum de 12 horas), critério das IV Diretrizes Brasileiras Sobre Dislipidemias e Prevenção da Ateroesclerose ${ }^{17}$. Concentrações de colesterol total, triglicérides e glicose determinadas usando um teste enzimático colorimétrico, com níveis de LDL calculados por aplicação da fórmula de Friedwald ${ }^{18,19}$ (para triglicérides $<400 \mathrm{mg} / \mathrm{dl}$ ); colesterol total: ótimo ( $\leq 200 \mathrm{mg} \%$ ), limítrofe $(200-239 \mathrm{mg} \%)$ e alto ( $\geq 240 \mathrm{mg} \%)$.; LDL colesterol: ótimo ( $<100 \mathrm{mg} \%)$, limítrofe (130-159 mg\%), alto (160-189 mg\%) e muito alto ( $\geq 190$ mg\%).; HDL colesterol: baixo (< $40 \mathrm{mg} \%$ ) e alto (>60 mg\%). Glicemia (jejum de 12 horas): elevada ou disglicemia (entre 100 e $125 \mathrm{mg} \%)$; diabete melito $(\geq 126 \mathrm{mg} \%)^{17}$. Insulinemia (jejum de 12 horas): através de quimioluminescência, expressa em $\mu \mathrm{U} / \mathrm{ml}$. Determinação da Proteína C-reativa (jejum de 12 horas): pelo método turbidimétrico, expressa em $\mathrm{mg} / \mathrm{l}$, com ponto de corte para definição de PCR elevada de $3 \mathrm{mg} / \mathrm{dl}^{20}$. Uricemia (jejum de 12 horas): elevada (>7 mg/dl para homens e $6 \mathrm{mg} / \mathrm{dl}$ para mulheres $\mathrm{s}^{21}$ ). Fibrinogênio no sangue (jejum de 12 horas): elevado (> $4 \mathrm{mg} / \mathrm{I}^{22}$ ).

Como variáveis independentes sócio-demográficas, foram considerados idade: igual ou superior a 20 anos; gênero: masculino ou feminino; situação conjugal: com ou sem companheiro(a); escolaridade, renda e número de moradores no domicílio; hábitos alimentares: uso de sal, consumo de gorduras, ingestão calórica; prática de atividade física: sedentarismo (definido quando o indivíduo relatou inatividade física no trabalho, no deslocamento até o trabalho e nos momentos de folga); hábito tabágico: fumante (pelo menos um cigarro por dia), ex-fumante (o que fumou regularmente no passado e abandonou completamente o tabagismo); hábito alcoólico: uso de bebida alcoólica (frequência, tipo e quantidade ingerida); história familiar: pais, ou parentes em primeiro grau, menores de 60 anos, com antecedentes de hipertensão, infarto do miocárdio ou AVE.

Para o gerenciamento e a análise dos dados, organizou-se um banco de dados utilizando-se os Programas Microsoft Office ACCESS 2003 e Epi Info 2000, versão 11.0, para análise exploratória, bivariada e estratificada, e o programa SPSS, versão 3.3.4, para a análise múltipla. Para o grupo total, estratificado por gênero, e para variáveis independentes, foram calculadas as taxas de prevalência de $S M$, os intervalos de confiança (IC) $95 \%$ e as medidas de associação. A associação entre as variáveis independentes e a SM foi testada por meio do teste qui-quadrado de Pearson. Para as variáveis contínuas, testou-se a hipótese de normalidade através do teste de KolmogorovSmirnov. As médias de variáveis com distribuição normal foram comparadas por meio do teste t-Student e para as que não apresentavam distribuição normal, aplicou-se o teste não paramétrico de Mann-Whitney. O nível de significância crítico adotado para a rejeição da hipótese nula foi de até $5 \%(p<$ $0,05)$. A análise múltipla foi feita através da Regressão Logística pelo método stepwise forward.

Este estudo foi autorizado após avaliação do Comitê de Ética em Pesquisa do Hospital Universitário Julio Muller (HUJM). Utilizou-se também um Termo de Consentimento Livre e Esclarecido, conforme Resolução 196/96 do Conselho Nacional de Saúde de 10 de outubro de 1996.

\section{Resultados}

Dos 120 participantes analisados, 60 eram mulheres. A média de idade da população estudada foi de 58,3 \pm 12,6 anos, sem diferença quanto ao gênero, faixa etária (adultos e idosos) e antecedente familiar de HA. A prevalência total de SM entre os hipertensos foi de $70,8 \%$, significativamente mais frequente entre as mulheres $(81,7 \%$ vs. $60 \%$; $\mathrm{RP}=1,36$; IC95\% 1,07-1,73). Do total, 15\% dos hipertensos apresentaram cinco critérios e $21,7 \%$ quatro critérios de SM (Figura 1). Sob a análise bivariada ocorreu uma associação significativa entre SM e gênero, antecedente familiar de HA, IMC superior a $25 \mathrm{~kg} / \mathrm{m}^{2}$, CC muito aumentada e RCQ aumentada. Não houve associação com outras variáveis sócio-demográficas, sedentarismo, tabagismo e hábitos alcoólico ou alimentar (Tabela 1).

Em relação às variáveis laboratoriais, observou-se uma associação entre SM e HDL-colesterol baixo, VLDL-colesterol elevado, ácido úrico elevado, e presença de resistência insulínica (Tabela 1).

Pela regressão múltipla observaram-se ORaj estatisticamente significantes para associações entre SM e IMC igual ou superior a $25 \mathrm{~kg} / \mathrm{m}^{2}$, resistência insulínica e presença de familiar em primeiro grau com HA (Tabela 2).

\section{Discussão}

Este trabalho compõe um projeto mais amplo, cuja finalidade foi descrever algumas características epidemiológicas associadas à Hipertensão Arterial na Região Centro-Oeste: em cidades de Goiás (Goiânia e Firminópolis) e de Mato Grosso (Cuiabá e Nobres). O objetivo deste estudo, de corte transversal, foi apresentar a análise de algumas características da síndrome metabólica entre hipertensos de Cuiabá - MT. A seleção amostral considerou a proporcionalidade da densidade populacional de cada macrozona urbana. Os dados foram obtidos por inquérito domiciliar, padronizado e previamente aplicado através de estudo piloto. Apesar de critérios bem definidos para o diagnóstico de SM, a diversidade de fatores causais dificulta a comparação da prevalência entre diferentes populações. Já em populações específicas, sua apresentação pode ocorrer com componentes individuais distintos ${ }^{23}$. Diferenças na distribuição por sexo, faixa etária, dieta, grau de atividade física, características étnicas e genéticas, podem afetar a proporção dos diversos componentes da SM. Assim, as prevalências encontradas neste estudo são representativas de uma população urbana, heterogênea, miscigenada quanto à ascendência, constituída de adultos com idade superior a 20 anos, da região Centro-Oeste do país.

Em um grupo específico de hipertensos, a SM, diagnosticada segundo os critérios tradicionais, conferiu um aumento do risco relativo em 2,64 vezes (IC95\% = $1,52-4,58)$ para o primeiro evento cardiovascular ${ }^{24}$. No presente estudo, em hipertensos de Cuiabá, observou-se prevalência geral de SM de $70,8 \%$, segundo os critérios do NCEP-ATP III, com predomínio significativo entre as mulheres. Estudos de prevalência de SM entre hipertensos são ainda escassos no Brasil. Em Salvador, Bahia, Bulhões 


\section{Artigo Original}

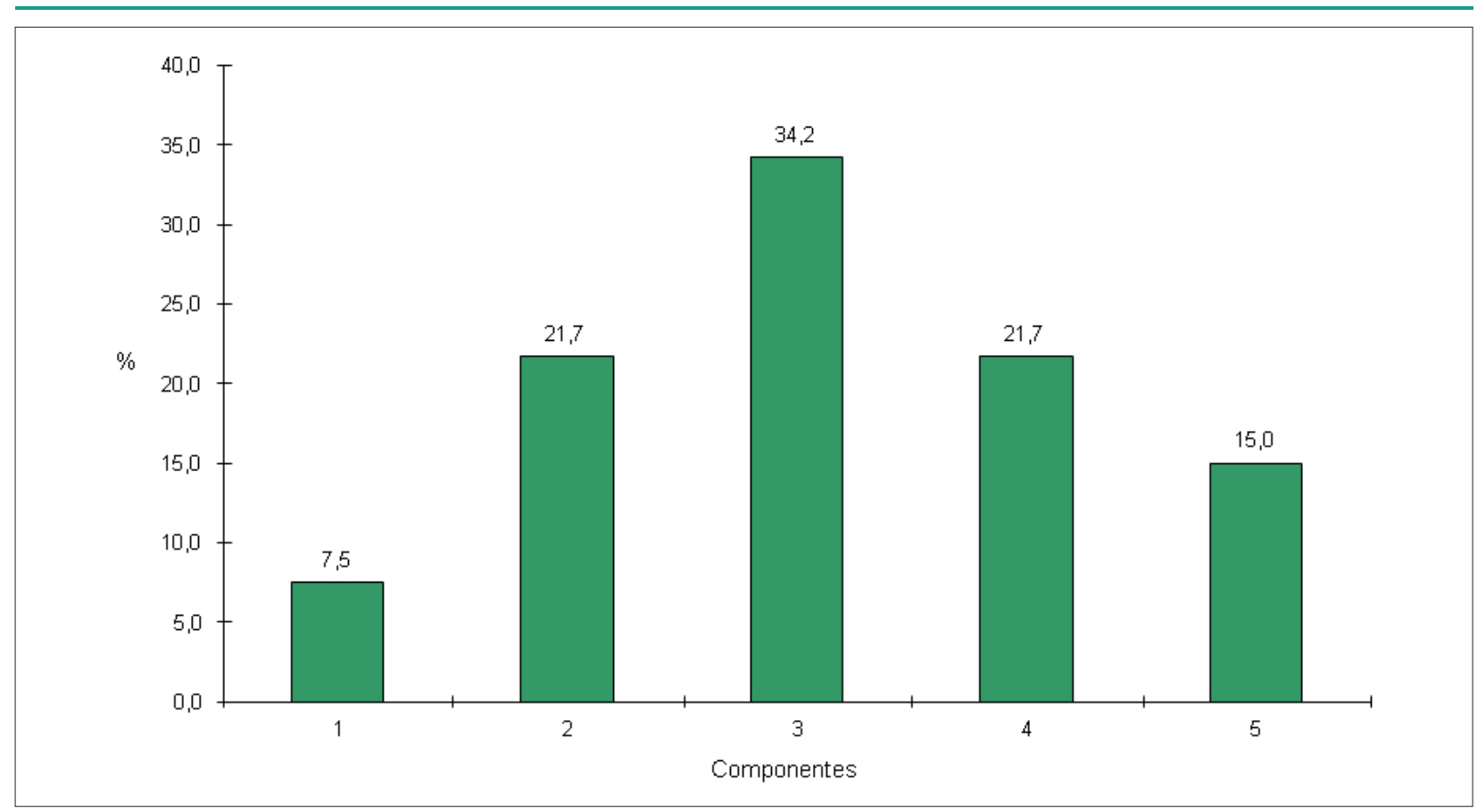

Fig. 1 - Distribuição do número de componentes de síndrome metabólica; Cuiabá - MT, 2007.

Tabela 1 - Prevalência de síndrome metabólica segundo variáveis sócio-demográficas, antropométricas e laboratoriais; Cuiabá-MT, 2007

\begin{tabular}{|c|c|c|c|c|}
\hline Variáveis (sim/não) & Amostra (\%) & SM presente (\%) & SM ausente (\%) & $\mathrm{p}$ \\
\hline Sexo (Masc/Fem) & $50,0 / 50,0$ & $60,0 / 81,7$ & $40,0 / 18,3$ & 0,009 \\
\hline Faixa Etária $<60 / \geq 60$ anos & $52,4 / 47,6$ & $71,4 / 70,2$ & $28,6 / 29,8$ & 0,880 \\
\hline Escolaridade $>8 / \leq 8$ anos & $63,3 / 36,7$ & $72,1 / 71,0$ & $28,9 / 29$ & 0,902 \\
\hline Sedentarismo & $28,3 / 71,7$ & $79,4 / 67,4$ & $20,6 / 32,6$ & 0,194 \\
\hline Hábito Alcoólico & $33,3 / 66,7$ & $72,5 / 70,0$ & $27,5 / 30,0$ & 0,776 \\
\hline Hábito Tabágico & $47,5 / 52,5$ & $64,9 / 76,2$ & $35,1 / 23,8$ & 0,204 \\
\hline Familiar com Hipertensão & $67,5 / 32,5$ & $80,2 / 51,3$ & $19,8 / 48,7$ & 0,001 \\
\hline $\operatorname{IMC}\left(\mathrm{kg} / \mathrm{m}^{2}\right)(<25 / \geq 25)$ & $30,8 / 69,2$ & $43,2 / 83,1$ & $56,8 / 16,9$ & $<0,001$ \\
\hline CC muito aumentada & $64,0 / 36,0$ & $92,2 / 46,4$ & $7,8 / 53,6$ & $<0,001$ \\
\hline RCQ aumentada & $55,8 / 44,2$ & $80,6 / 58,5$ & $29,4 / 41,5$ & 0,008 \\
\hline Colesterol total elevado & $22,5 / 77,5$ & $81,5 / 67,7$ & $18,5 / 32,3$ & 0,167 \\
\hline HDL baixo & $63,3 / 36,7$ & $78,9 / 56,8$ & $21,1 / 43,2$ & 0,010 \\
\hline LDL elevado & $24,2 / 75,8$ & $69,0 / 71,4$ & $31,0 / 28,6$ & 0,799 \\
\hline VLDL elevado & $29,2 / 70,8$ & $97,1 / 60,0$ & $2,9 / 40,0$ & $<0,001$ \\
\hline Ácido Úrico elevado o & $14,2 / 85,8$ & $94,1 / 67,0$ & $5,9 / 33$ & 0,016 \\
\hline PCR elevada & $5,8 / 94,2$ & $71,4 / 70,8$ & $28,6 / 29,2$ & 0,971 \\
\hline Fibrinogênio elevado & $1,7 / 98,3$ & $100,0 / 70,3$ & $0,0 / 29,7$ & 0,840 \\
\hline HOMA $>2,71$ & $60,8 / 39,2$ & $86,3 / 46,8$ & $13,7 / 53,2$ & $<0,001$ \\
\hline
\end{tabular}

e Araújo ${ }^{25}$ estudaram 102 hipertensos maiores de 18 anos, atendidos em hospital-escola, registrando uma prevalência de SM de 71,6\% (critérios do NCEP-ATP III). O resultado foi similar ao obtido no presente estudo. Apesar de diferentes proporções entre os gêneros, e do estudo de Salvador ter avaliado a demanda espontânea de um hospital-escola, observou-se uma semelhança quanto à idade e IMC médios e a frequência de cinco critérios (NCEP-ATP III) nos dois estudos (17,9\% em Salvador, 15\% em Cuiabá).

A predominância de SM entre as mulheres $(81,7 \%$ vs $60,0 \% ; p=0,009)$, sem diferença entre adultos e idosos, concorda com o estudo da população rural do Vale do 
Tabela 2 - Resultados da análise múltipla para as características associadas à síndrome metabólica em pacientes hipertensos; Cuiabá - MT, 2007

\begin{tabular}{lccc}
\hline Variáveis & Odds Ratio & IC $95 \%$ & $p$ \\
\hline IMC $\geq 25 \mathrm{~kg} / \mathrm{m}^{2}$ & 5,61 & $1,92-16,42$ & 0,014 \\
\hline HOMA $>2,71$ & 9,85 & $3,23-30,05$ & 0,001 \\
\hline Familiar com HAS & 4,15 & $1,33-13,03$ & 0,002 \\
\hline
\end{tabular}

Jequitinhonha - MG (33,6\% vs. 7,7\%), com prevalência geral de $21,6 \%{ }^{26}$ e com outro estudo no semi-árido baiano ${ }^{27}$, em faixa etária igual ou superior a 45 anos. O predomínio de SM nesta faixa etária, possivelmente por influência do climatério, pode justificar a rapidez da progressão de eventos cardiovasculares em mulheres com SM após o climatério ${ }^{28}$. Contrariamente, em adultos americanos (prevalência geral de 23,7\% - NCEP-ATP III), observou-se o incremento de SM com o aumento da idade, sem diferença entre os gêneros ${ }^{29}$, em concordância com o estudo de base populacional em adultos de Vitória-ES (prevalência geral de 29,8\%), onde também não ocorreu diferença entre os gêneros ${ }^{23}$. Diferentemente, o Estudo SESI, entre trabalhadores da indústria de cinco estados brasileiros, revelou prevalência geral de SM de 10,5\%, significativamente maior nos homens $(11,5 \% \text { vs. } 8,1 \%)^{30}$.

Não foi observada uma associação entre a baixa escolaridade e a SM, em concordância com os resultados do Estudo $\mathrm{SESI}^{28}$ em trabalhadores da indústria e em hipertensos de Goiânia-GO ${ }^{31}$. Esse fato pode estar relacionado com o avanço significativo da obesidade em, praticamente, todas as faixas etárias e níveis sociais, em especial entre indivíduos de baixa renda e escolaridade. Não foi estabelecida uma associação entre a renda per capita e a SM, contrariamente ao observado em Vitória-ES, onde mulheres da classe $\mathrm{A}$ apresentaram menor prevalência de SM $(17,9 \%$ vs $38,7 \%)$ em comparação às das classes D e $\mathrm{E}$, de menor renda ${ }^{23}$.

Outras variáveis relacionadas ao estilo de vida, tais como: sedentarismo, horas assistindo televisão e hábitos tabágicos, alcoólicos e alimentares (ex.: retirar a gordura da carne e a pele do frango ou utilizar sal adicional à mesa) não mostraram associação com a SM no presente estudo, mediante análise de regressão múltipla. Velasquez-Melendez e cols. ${ }^{26}$ também não observaram associação entre o tabagismo e a SM. A falta da avaliação quantitativa da ingestão de etanol e sal nos participantes do presente estudo, pode ter influenciado estes resultados.

Em relação à associação entre a SM e a resistência insulínica, Bulhões e Araújo $^{25}$ observaram valores de insulinemia de jejum significativamente maiores entre os indivíduos portadores de SM $(p=0,006)$. No presente estudo, de acordo com os critérios adotados ${ }^{16}$, verificouse que mais de $80 \%$ dos hipertensos portadores de SM apresentaram índice HOMA superior a 2,71, em concordância com outros estudos acerca do relevante papel da resistência insulínica na fisiopatogenia da $\mathrm{SM}^{32}$. O fato do índice HOMA permanecer associado à SM sob análise multivariada, aponta para a possibilidade de utilizar-se este índice como indicador de resistência à insulina em portadores de SM, com evidente repercussão na terapêutica e no prognóstico, em especial dos não diabéticos ${ }^{33,34}$. Neste estudo, observou-se expressiva e significativa prevalência de CC aumentada e muito aumentada nos portadores de SM, em especial entre as mulheres. O IMC médio foi significativamente maior nos portadores de $S M$, sendo que valores acima de $25 \mathrm{~kg} / \mathrm{m}^{2}$ exerceram importante influência sobre a variável-desfecho $(p=0,014)$, em concordância com Oliveira e cols. ${ }^{20}$, e contrariamente a Bulhões e Araújo $^{25}$. Este resultado reforça o elo fisiopatológico entre a adiposidade central e a resistência insulínica ${ }^{34}$. Quanto à relação entre o estado inflamatório e a SM, não foi detectada uma associação significativa entre valores de PCR e SM, ou resistência insulínica. Este aspecto pode ser atribuído ao fato da SM e a hipertensão serem, ambas, síndromes vasculares inflamatórias ${ }^{35}$ ou pela técnica utilizada (turbidimetria). A determinação da PCR ultra-sensível por outro método (imunonefelometria hipersensível), poderia estabelecer resultados mais precisos. A fração LDLcolesterol, marcadamente a fração pequena e densa, apesar de não compor os critérios diagnósticos da SM, apresenta importante associação com a resistência insuflínica e a SM ${ }^{3}$. Em relação às frações lipídicas, observou-se neste estudo uma associação positiva e significativa entre o VLDL elevado e a SM, e uma negativa com níveis elevados de colesterol total (>240 mg\%) e LDL-colesterol (>160 mg\%), em acordo com Oliveira e cols. ${ }^{27}$.

Em relação aos antecedentes familiares, observou-se neste estudo uma associação positiva entre a SM e a presença de familiar em primeiro grau com hipertensão, revelado por análise de regressão múltipla $(p=0,002)$. Este original achado chama a atenção para os resultados de alguns estudos, que abordaram a importância do fator genético, e de um elo molecular unificador da fisiopatologia da $\mathrm{SM}^{32}$. Já $\mathrm{WU}$ e cols. ${ }^{36}$ demonstraram que a região próxima ao gene da lipoproteína lípase (gene $L P L$ ), no braço curto do cromossomo 8, influencia as variações da PAS em membros de famílias não diabéticas que apresentaram risco substancialmente elevado para desenvolver resistência à insulina e diabete melito $2(\mathrm{SM})^{36}$. Por outro lado, Cheng e cols. ${ }^{37}$, estudando 390 membros de famílias hispânicas de hipertensos, observaram uma ligação coincidente entre a insulina de jejum e a PA no braço longo do cromossomo 7, demonstrando um determinismo genético importante para componentes da SM localizado nesse cromossoma ${ }^{37}$. A pesquisa de polimorfismos do gene SA, realizada por Iwai e cols. ${ }^{38} \mathrm{em} 4$ mil indivíduos representantes da população geral do Japão, mostrou uma associação entre o gene SA, a hipertrigliceridemia, a hipercolesterolemia, a obesidade e a hipertensão, reforçando a hipótese de base genética comum para a $\mathrm{SM}^{38}$. Em seu estudo, Love-Gregory e cols. $^{39}$ encontraram uma associação específica entre o gene CD36 do cromossomo 7, a SM e o metabolismo do HDL-colesterol. O grupo do presente estudo revelou que hipertensos de Cuiabá, com antecedentes familiares de AVE, apresentaram risco 1,7 vez maior de hipertensão em 
comparação aos que não apresentavam tais antecedentes ${ }^{40}$. Estas investigações, realizadas em populações diversas e em diferentes regiões do mundo, revelam a grande dificuldade para o entendimento das bases genéticas da SM.

Este estudo revelou uma elevada prevalência de SM entre os hipertensos de Cuiabá, e sua significativa associação com o IMC superior a $25 \mathrm{~kg} / \mathrm{m}^{2}$, a resistência insulínica (avaliada pelo índice HOMA) e, em especial, a história familiar de hipertensão. Estes resultados sugerem o aprofundamento deste assunto através de novos estudos epidemiológicos, preferencialmente de base populacional e com avaliação de componente(s) genético(s).

\section{Potencial Conflito de Interesses}

Declaro não haver conflito de interesses pertinentes.

\section{Fontes de Financiamento}

O presente estudo não teve fontes de financiamento externas.

\section{Vinculação Acadêmica}

Este artigo é parte de tese de mestrado de Gilberto Paulo Pereira Franco pelo Instituto de Saúde Coletiva da Universidade Federal de Mato Grosso.

\section{Referências}

1. Reaven GM. Banting Lecture 1988. Role of insulin resistance in human disease. Diabetes. 1988; 37 (12): 1595-607.

2. Lopes HF. Síndrome metabólica: aspectos históricos, prevalência, morbidade e mortalidade. Rev Soc Cardiol Estado de São Paulo. 2004; 14 (4): 539-43.

3. Sociedade Brasileira de Cardiologia. I Diretriz Brasileira de diagnóstico e tratamento da síndrome metabólica. Arq Bras Cardiol. 2005; 84 (sup 1): 1-28.

4. Lopes HF, Egan BM. Desequilíbrio autonômico e síndrome metabólica: parceiros patológicos em uma pandemia global emergente. Arq Bras Cardiol. 2006; 87: 538-47.

5. He J, Muntner P, Chen J, Roccella EJ, Streiffer RH, Whelton PK. Factors associated with hypertension control in the general population of the United States. Arch Intern Med. 2002; 162: 1051-8.

6. Kannel WB. Risk stratification in hypertension: new insights from the Framingham Study. Am J Hypertens. 2007; 13: 3S-10S.

7. Ozsahin AK, Gokcel A, Sezgin N, Akbaba M, Guvener N, Ozisik L, et al. Prevalence of the metabolic syndrome in a Turkish adult population. Diabetes Nutr Metab. 2004; 17: 230-4.

8. Gu D, Reynolds K, Wu X, Chen J, Duan X, Whelton PK, et al, for the InterA-S/A Clollaborative Group. Prevalence of the metabolic syndrome and overweight among adults in China. Lancet. 2005; 356: 1398-405.I

9. Ford ES, Giles WH, Ditez WH. Prevalence of the metabolic syndrome among US adults: findings from the third National Health and Nutrition Examination Survey. JAMA. 2002; 287: 356-9.

10. Ford ES. Prevalence of metabolic syndrome defined by the International Diabetes Federation among adults in the U.S. Diabetes Care. 2005; 28 : 2745-9.

11. Zimmet PZ, Kelly West Lecture 1991. Challenges in diabetes epidemiology from West to the rest. Diabetes Care. 1992; 15: 232-52.

12. Cassanelli T. Prevalência de hipertensão arterial sistêmica e fatores associados na região urbana de Cuiabá: estudo de base populacional. [Dissertação]. Cuiabá-MT: Instituto de Saúde Coletiva da Universidade Federal de Mato Grosso; 2005.

13. Sociedade Brasileira de Cardiologia. V Diretrizes Brasileiras de hipertensão arterial. Arq Bras Cardiol. 2007; 89 (3): e24-e79.

14. O'Brien E, Mee E, Atkins N, Thomas M. Evaluation of three devices for selfmeasurement of blood pressure according to the revised British Hypertension Society Protocol: the Omron Hem-705Cp, Phillips HP5332, and Nissei DS175. Blood Press Monit. 1996; 1: 1-7.

15. Obesity: preventing and managing the global epidemic. Geneva: World Health Organization; 1998.

16. Geloneze B, Tambascia MA. Avaliação laboratorial e diagnóstico da resistência insulínica. Arq Bras Endocrinol Metab. 2006; 50 (2): 208-15.
17. Sociedade Brasileira de Cardiologia. IV Diretriz Brasileira sobre dislipidemias e prevenção da aterosclerose. Arq Bras Cardiol. 2007; 88 (sup 1): 2-19.

18. Third Report of the National Cholesterol Education (NCEP) Expert Panel on Detection, Evaluation, and Treatment of high blood cholesterol in adults (Adult Treatment Panel III) final report. National Cholesterol Education Program (NCEP) Expert Panel on Detection, Evaluation, and Treatment of High Blood Cholesterol in adults (Adult Treatment Panel III). Circulation. 2002; 106: 3143-421.

19. Friedwald WT, Levy AI, Frederickson DS. Estimation of concentrations of low density cholesterol in plasma, without use of the preparative ultracentrifuge. Clin Chem. 1972; 18: 499-502.

20. Maduro IPNN, Albuquerque FM, Nonino CB, Borges RM, Marchini JS. Hiperuricemia em obesas sob dieta altamente restritiva. Arq Bras Endocrinol Metab. 2003; 47 (3): 266-70.

21. Malik S, Wong ND, Franklin S, Pio J, Fairchild C, Chen R. Cardiovascular disease in U.S. patients with metabolic syndrome, diabetes, and elevated C-reactive protein. Diabetes Care. 2005; 28: 690-3.

22. Moura PA, Silva Torres VI, Marreiro DN, Lobão A, Souza SSR, Mendes Filho JL. Concentrações séricas de fibrinogênio e proteína $C$ reativa como biomarcadores inflamatórios na predição de risco da doença aterosclerótica em pacientes com sobrepeso. Rev Bras Nutr Clin. 2006; 21 (4): 290-5.

23. Salaroli LB, Barbosa GC, Mill JG, Molina, CB. Prevalência de síndrome metabólica em estudo de base populacional, Vitória, Espírito Santo, Brasil. Arq Bras Endocrinol Metab. 2007: 51 (7): 1143-52.

24. Pierdomenico SD, Lapena D, Di Tommazo R, Di Carlo S, Caldarella MP, Neri $\mathrm{M}$, et al. Prognostic relevance of metabolic syndrome in hypertensive patients at low-to-medium Risk. Am J Hypertens. 2007; 20 (12): 1291-6.

25. Bulhões K, Araújo L. Metabolic syndrome in hypertensive patients: correlation between antropometric data and laboratory findings. Diabetes Care. 2007; 30: 1624-6.

26. Velasquez-Melendez G, Gazzinelli A, Correa-Oliveira R, Pimenta AM, Kac G. Prevalência de síndrome metabólica em área rural do Brasil. São Paulo Med J. 2007; 125 (3): 155-62.

27. Oliveira EP, Souza MLA, Lima MDA. Prevalência de síndrome metabólica em uma área rural do semi-árido baiano. Arq Bras Endocrinol Metab. 2006; 50 (3): 456-65.

28. Carr MC. The emergency of the metabolic syndrome with menopause. J Clin Endocrinol Metab. 2003; 88 (6): 2404-11.

29. Ford ES, Giles WH, Dietz WH. Prevalence of the metabolic syndrome aomong US adults: findings from the third National Health and Nutrition Examination Survey. JAMA. 2002; 287: 356-9.

30. Estudo SESI. Serviço Social da Indústria. Departamento Nacional. Perfil epidemiológico de fatores de risco para doenças não transmissíveis em 
trabalhadores da Indústria no Brasil. Brasília: SESI/DN, 2007.

31. Jardim PCBV, Gondim MRP, Monego ET, Moreira HG, Vitorino PVO, Souza WSKB, et al. Hipertensão arterial e alguns fatores de risco em uma capital brasileira. Arq Bras Cardiol. 2007; 88 (4): 452-7.

32. Grunfeld B, Balzareti M, Romo M, Gimenez M, Gutman R. Hyperinsulinemia in normotensive offspring of hypertensive parents. Hypertension. 1994; 23 : $112-5$.

33. Reaven GM. Metabolic syndrome: pathophysiology and implications for management of cardiovascular disease. Circulation. 2002; 106: 286-8.

34. Spósito AC, Santos RD, Ramires JAF. Avaliação do risco cardiovascular no excesso de peso e obesidade. Arq Bras Cardiol. 2002; 78 (supl 1): 3-13.

35. Björntorp P, Rosmond R. The metabolic syndrome - a neuroendocrine disorder? Br J Nutr. 2000; 83 (Suppl I): 549-57.

36. Wu DA, Bu X, Warden $\mathrm{CH}$, Shen DD, Jeng CY, Sheu WH, et al. Quantitative trait locus mapping of human blood pressure to a genetic region at or near the lipoprotein lipase gene locus on chromosome 8p22. J Clin Invest. 1996; 97: 2111-8.

37. Cheng LS, Davis RC, Raffel LJ, Xiang AH, Wang N, Quinones M, et al. Coincident linkage of fasting plasma insulin and blood pressure to chromosome 7q in hypertensive families. Circulation. 2001; 104: 125560

38. Iwai N, Katsuya T, Mannami T, Higaki J, Ogihara T, Kokame K, et al. Association between $\mathrm{SAH}$, an acyl-CoA synthetase gene, and hypertriglyceridemia, obesity, and hypertension. Circulation. 2002; 105: 41-7.

39. Love-Gregory L, Sherva R, Sun L, Wasson J, Schappe T, Doria A, et al. Variants in the CD36 gene associate with the metabolic syndrome and high-density lipoprotein cholesterol. Hum Mol Genet. 2008; 17 (11): 1695-704.

40. Araújo J, Salerno HD, Scala LCN. Prevalence and epidemiological profile of hypertension in the adult population of the family health program in Cuiabá, Mato Grosso, Brazil. In: 20th Scientific Meeting of the International Society of Hypertension; São Paulo; 2004. Hypertension. 2004; 22: S107. 